\title{
Multi-author Review
}

Medium- and short-chain dehydrogenase/reductase gene and protein families

Coordinator: H. Jörnvall 



\title{
MDR and SDR gene and protein superfamilies
}

\author{
H. Jörnvall \\ Department of Medical Biochemistry and Biophysics, Karolinska Institutet, 17177 Stockholm (Sweden), \\ Fax: +468337 462, e-mail: Hans.Jornvall@ki.se \\ Online First 14 November 2008
}

\section{Introduction}

Alcohol dehydrogenases (ADHs) are founding members of the medium-chain and short-chain dehydrogenase/reductase (MDR and SDR) gene and protein superfamilies. They have a long history (yeast ADH purified in 1937, the first mammalian form in 1948), and the superfamilies have contributed important steps to our knowledge of protein evolution, enzymatic mechanisms, metalloproteins, metabolic functions, and regulatory roles. They still supply surprising discoveries and novel functions.

Enzymatically, $\mathrm{ADH}$ is at the base of the Enzyme Commission nomenclature (EC 1.1.1.1). It is also an activity which, like that of many dehydrogenases, shows extensive multiplicity, including chemically modified sub-forms, isozymes, classes, and separate enzymes, therefore exhibiting redundancy with overlaps in activity. Hence, functional assignments in this field are not always easy. It is clear that ADHs constitute a part of our defense system against many reactive compounds. As such, they functionally resemble cytochrome P450 s, but with the added advantage that they do not leave reactive oxygen species which can hurt the tissues. True, many of the aldehydes formed are reactive and dangerous, but we have an effective battery of aldehyde dehydrogenases, and combined, many of the MDR, SDR, aldehyde and other dehydrogenases form integrated metabolic pathways.

MDR and SDR superfamilies are large. In humans they are estimated to have minimally $25 \mathrm{MDR}$ genes (review 1) and well over double that number of SDR genes (review 2). In fact, the SDR superfamily in present gene summaries appears size-wise to be among the top ten and has been mentioned as the third-largest family in some living systems investigated. Mechanistically, it is also very versatile (reviews 2 and 4), and its gene products cover at least half of the six classes of activity that enzymes can be functionally divided into (i.e. oxidoreductases, lyases and isomerases). Furthermore, the ancestral building blocks constitute central scaffolds in numerous additional enzymes. Nature appears to be able to use the MDR and SDR basic folds for positioning separate active sites and residues in different combinations, meaning very few critical residues or absolutely conserved sites. The present series of reviews (reviews $1-4$ ), demonstrate many defined functions in the families, but also some uncertainties regarding mechanistic relationships or functional roles.

Metalloenzyme-wise, several MDR forms have active-site zinc, and sometimes even a second site with a 'structural' zinc, both however non-obligatory in other enzymes within the families, again demonstrating a basic scaffold but non-conservation of details. In relation to the metalloenzyme aspects (review 7), we have a study of a peptide model of an ADH metal site as an adjacent research paper [1]. It shows a switch of metal ligands, with further implications. Notably, the structures of the real enzymes may switch too, in response to an additional liganding residue, and these relationships differ between classes, between subunits in the protein, and between the forms with and without coenzyme (reviews 3 and 7). In short, the metal sites in the zinc-dependent MDR enzymes illustrate complex relationships.

Repeated steps of enzymogenesis are detailed in the two reviews of the family relationships (reviews 1 and 2 ), and have been well studied in the evolution of the MDR-ADH classes from an ancestral formaldehyde dehydrogenase. Perhaps, we should also mention two further functional aspects: one, that MDR-ADH is still the enzyme metabolizing the ethanol we drink. As such, it is medically important, and has isozyme differences that explain population differences in alcohol breakdown. This and other alcoholism-related aspects are not treated here, but have been treated at 
other occasions (for example [2] from the same publisher). The second special aspect is that activations/inactivations of steroid hormones (review 2), and participation in retinoid (review 5) and possibly nitric oxide metabolism (review 6) give SDR and MDR regulatory roles.

A few words about the methods and the authors. Our lives with these enzymes, although covering only one generation, have witnessed a joyful ride with impressive developments in methods, as seen everywhere in molecular science. Initial traces of the relationships now treated were first touched upon when sequence analysis was at the protein level, alignments were assembled initially in yearly printed issues (Atlas of Protein Sequence and Structure by Margaret Dayhoff), interpretations of 3D structures meant building models and using big plates with electron density maps, phylogenetic studies of dehydrogenases meant cycling to a department of numerical analyses to get access to a computer, and family sizes were graspable. Now, genome sequencing, recombinant protein analysis, gene knockout studies, computerization throughout, and extensive databases available for everyone have created the present scene, but as seen in the reviews, functional assignments and mechanistic conclusions are still difficult.

When being invited to edit a multi-author review on MDR/SDR dehydrogenases, I started by asking several key players to take part. True, some are deceased and many others also not represented here. But I am grateful to all who have joined in this review series. Several of you are long-term collaborators. Together, we have seen the evolution of life sciences and can summarize one of the active research fields of the future - the role and control of 100 human MDR and SDR genes, literally hundreds of corresponding human MDR and SDR enzymes in disease characterization, monitoring and treatment, and tens of thousands of forms in understanding life and proteins, and for use in biotechnology and medicine.

Stockholm, 30 June 2008

Hans Jörnvall

\section{Content}

Review 1. Persson, Hedlund and Jörnvall: MDR proteins

Review 2. Kavanagh, Jörnvall, Persson and Oppermann: Diversity of short-chain dehydrogenases/reductases

Review 3. Eklund and Ramaswamy: Structures of MDR alcohol dehydrogenases

Review 4. Ladenstein, Winberg and Benach: Short-chain alcohol dehydrogenases

Review 5. Parés, Farrés, Kedishvili and Duester: MDRs and SDRs in retinoid metabolism

Review 6. Staab, Hellgren and Höög: Dual functions of ADH3

Review 7. Auld and Bergman: Zinc in alcohol dehydrogenase 


\section{Postscript on nomenclature}

Some words on nomenclature are relevant in relation to MDRs, ADHs, and the whole concept of superfamilies, families, classes, and isozymes. Over time, these expressions have had different meanings. Initially, when relationships were not known, other nomenclatures were often used. For outsiders, conversion of names is not always easy. Therefore, we present some explanations below, as well as the conventions presently aimed for.

\section{MDR.}

Initially, the MDR proteins were distinguished from SDR proteins [3], and since they differed in chain length (typically $\sim 350$ versus $\sim 250$ residues), they were then called long-chain and short-chain DHs. With the realization that there are further DHs with still longer chains, the medium-chain concept for the MDR proteins became accepted, and has now been prevalent for decades. MDR and SDR are the recognized superfamily names.

\section{Superfamily.}

MDRs and SDRs have extensive gene multiplicity, proteins of wide activity spreads, and extremes that differ far beyond conventional family relationships. Hence, the proper names for MDRs and SDRs should be superfamilies. We have tried to follow these names in the reviews. Nevertheless, just 'families' in short is still often used, even here, and suffices when the overall concepts are anyway clear.

\section{Family.}

The separate families correspond to recognizable functions. Often, the same type of activity (like $\mathrm{ADH}$ ) may occur in both superfamilies, and when it is desirable to emphasize this, these two families then become MDR-ADH and SDR-ADH, respectively. Of course, these two ADH families differ substantially, since MDR and SDR are separate superfamilies. MDR-ADH is the family of liver ADH enzymes, SDR-ADH the Drosophila ADH family. This parallel evolution of the same activity in different lines with repeated enzymogenesis is typical of many dehydrogenases.

\section{Classes.}

This is the stage of multiple enzymes derived from gene duplications at times in between the more distant family formations and the more recent isozyme formations. Typically, as with MDR-ADHs, isozymes have sequence identities above $90 \%$, classes identities at the $60 \%$ level, and families at the $25 \%$ level or there about. MDR-ADH has evolved from all these stages, and in humans has five classes, in vertebrates at least eight. All gene loci for ADH classes in humans occur as one gene cluster. The classes are functionally distinct and with considerable differences, especially for class III (also called glutathione-dependent formaldehyde dehydrogenase) and all non-III classes. The common liver ADH is of the class I type. Recognition of the classes allows distinction of orthologous from paralogous forms in species comparisons. However, it also creates two problems nomenclature-wise.

A small problem is that some classes, originally defined for separate species, may not be true classes, but simply species variants in rapidly evolving classes. This appears to be the case with vertebrate $\mathrm{ADH}$ classes V and VI. A big problem is that gene numbers have been given an order not based on the class numbering system, while protein nomenclature has followed the classes. Hence, as proteins the human ADH classes II-V are called by these numbers (in Arabic numerals) or special designations, but by another number as genes. Hopefully, gene nomenclatures can convert to the class nomenclature system, as previously suggested [4]. It will then be possible even for outsiders to correlate function (the classes) with structures (the genes) by identical numbers independent of species.

\section{Isozymes.}

MDR-ADHs are rich in isozymes, so recently formed that they often differ among species. The subunits of these isozymes were initially given letters based on function or alphabetical order. Nearly all early ADH characterizations, and those in the present reviews not further defined, concern the horse EE isozyme, and the human B1B1 (previously called $\beta_{1} \beta_{1}$ ) isozyme, both class I. The horse ADH subunits were the first isozyme differences studied, while the human ADH B1 and B2 subunits correspond to different alleloforms and constitute the well-known difference in ADHs between Caucasian and Oriental populations.

A final nomenclature problem for outsiders is the fact that the gene cluster corresponding to the $\mathrm{ADH}$ classes referred to above, also includes isozyme genes. Hence, we have a gene cluster with recently derived class I isozyme genes, and more distantly derived genes for the classes, together seven genes in one cluster on chromosome 4 (Fig. 3 in review 1). This is a further reason why we suggest use of the class-based nomenclature system [4]. 
1 Bergman, T., Zhang, K., Palmberg, C., Jörnvall, H. and Auld D. S. (2008) Zinc binding to peptide analogs of the structural zinc site in alcohol dehydrogenase: implications for an entatic state. Cell. Mol. Life Sci., this issue.

2 Jansson, B., Jörnvall, H., Rydberg, U., Terenius, L. and Vallee, B. L., eds. (1994) Toward a Molecular Basis of Alcohol Use and Abuse. Birkhäuser, Basel.

3 Jörnvall, H., Persson, M. and Jeffery, J. (1981) Alcohol and polyol dehydrogenases are both divided into two protein types, and structural properties cross-relate the different enzyme activities within each type. Proc. Natl. Acad. Sci. USA 78, 42264230.

4 Duester, G., Farrés, J., Felder, M. R., Holmes, R. S., Höög, J.-O., Parés, X., Plapp, B. V., Yin, S.-J. and Jörnvall, H. (1999) Recommended nomenclature for the vertebrate alcohol dehydrogenase gene family. Biochem. Pharmacol. 58, 389-395.

To access this journal online: http://www.birkhauser.ch/CMLS 